\title{
A Free Boundary Problem for a Leslie-Gower Predator-Prey Model in Higher Dimensions and Heterogeneous Environment
}

\author{
Shiwen Niu, Hongmei Cheng* \\ School of Mathematics and Statistics, Shandong Normal University, Jinan, P. R. China
}

Email address:

hmcheng@sdnu.edu.cn (H. Cheng)

${ }^{*}$ Corresponding author

To cite this article:

Shiwen Niu, Hongmei Cheng. A Free Boundary Problem for a Leslie-Gower Predator-Prey Model in Higher Dimensions and Heterogeneous Environment. American Journal of Applied Mathematics. Vol. 8, No. 5, 2020, pp. 284-292. doi: 10.11648/j.ajam.20200805.17

Received: September 14, 2020; Accepted: October 12, 2020; Published: October 26, 2020

\begin{abstract}
This paper is mainly concerned with some free boundary problems for a modified Leslie-Gower predator-prey model in higher dimensional and heterogeneous environment. To keep it simple in this article, we assume that the environment and solutions are all radially symmetric. We consider the problem which be used to describe the spreading of an introduced predator species in higher dimensional and heterogeneous environment. We will assume that the prey is initially uniformly well disturbed. The prey undergoes the diffusion and growth in the entire space $\mathbb{R}^{n}$. The predator is initially introduced in some localized location. We establish that a spreading-vanishing dichotomy is held for this model. We use the comparison principle. we will give the existence, uniqueness and some estimates of the solution to the problem. We study the asymptotic behavior of two species evolving. The free boundary represents the spreading front of the predator species. The boundary condition is described by classic Stefan-like condition. It is proved that the problem addressed is well posed, and that the predator species disperses to all domains in finite time. The long time behaviors of solution and criteria for spreading and vanishing of predator species are also provided. Furthermore, in the case that spreading of predator species happens, we deduce some rough estimates of the spreading speed.
\end{abstract}

Keywords: Free Boundary, Predator-Prey Model, Spreading-Vanishing Dichotomy, Spreading Speed, Heterogeneous Environment

\section{Introduction}

It is an important issue how to understand the nature and spreading of an invasive species in mathematical ecology. In recent years, many mathematicians have established various invasion models and investigated them from the viewpoint of mathematical ecology, refer to [2, 3, 26, 28, 29, 30] etc. For the most theoretical approaches, they are all based on or started with single species models. In order to realize the spreading mechanism of a new or invasive species, Du and Lin [13] deduced the following free boundary problem of the diffusive logistic equation

$$
\begin{cases}u_{t}-d u_{x x}=u(a-b u), & t>0,0<x<h(t) \\ u(t, h(t))=0, u_{x}(t, 0)=0, & t>0 \\ h^{\prime}(t)=-\mu u_{x}(t, h(t)), & t>0 \\ h(0)=h_{0}, u(0, x)=u_{0}(x), & 0 \leq x \leq h_{0}\end{cases}
$$

where $x=h(t)$ is the moving boundary to be determined, $a$, $b, d$ are given positive constants, $h_{0}>0$ denotes the size of initial habitat, $\mu>0$ is the ratio of expanding speed of the free boundary and population gradient at expanding front, and $u_{0}$ is an given positive initial function. They have obtained the spreading-vanishing dichotomy result.

Since then, the problems describing the spread by free boundary have been studied intensively, such as in $[1,4,16$, 21, 22, 37]. As an example, Kaneko and Yamada in [24] studied the free boundary problem which condition $u_{x}=0$ at $x=0$ in (1) is replaced by $u=0$. Du, Guo and Peng in [10], and $\mathrm{Du}$ and Liang in [12] considered the free boundary problem in the time periodic environment. Peng and Zhao in [31] studied the seasonal succession case. When the nonlinear term $u(a-b u)$ is replaced by a general function 
$f(u)$, this problem has been investigated by Du and Lou in [15] and Du, Matsuzawa and Zhou in [17]. For the population model, there are many authors who have studied the diffusive competition system with the free boundary, such as Du and Lin in [14], Guo and $\mathrm{Wu}$ in [19, 20], Wang in [32, 33, 34], wang and Zhang in [35], wang and Zhao in [36], etc. For the initial value problem of the Leslie-Gower predatorprey model, A. Ducrot in [18] has studied some spreading properties of modified Leslie-Gower predator-prey reactiondiffusion system. In [6], we have considered the spreading speed properties for the Leslie-Gower predator-prey model with the fractional diffusion term $\Delta^{\alpha}(\alpha \in(0,1))$. In [7], we have showed the existence and stability of the Leslie-Gower predator-prey model with nonlocal diffusion. Liu et al. in [27] obtained the asymptotic behavior of two species evolving in a domain with a free boundary in onedimensional environment.
In consideration of the environment heterogeneity, Du and Guo in $[8,9]$ have studied the diffusive logistic model with a free boundary in heterogeneous environment, where the heterogeneous environment coefficients were required to have positive lower and upper bounds. They also obtained the corresponding spreading-vanishing dichotomy results. The predator-prey systems with heterogeneous environment have also been examined extensively, refer to [11, 39]. For instance, Wang and Zhao in [38] gave the discussion of the competition model with free boundary in the higher dimensional and heterogeneous environment. Some epidemic models with free boundary have been considered by some authors, such as in [23].

Motivated by those results, we consider in this paper with the following Leslie-Gower predator-prey reaction-diffusion system with free boundary and radial symmetry

$$
\begin{cases}u_{t}-d \Delta_{r} u=u(a(r)-b(r) u)-c(r) u v, & t>0, r>0, \\ v_{t}-\Delta_{r} v=v\left(\alpha(r)-\frac{\beta(r) v}{u+\delta}\right), & t>0,0<r<h(t), \\ v(t, r)=0, & t>0, r \geq h(t), \\ u_{r}(t, 0)=v_{r}(t, 0)=0, h^{\prime}(t)=-\mu v_{r}(t, h(t)), & t>0, \\ h(0)=h_{0}, v(0, r)=v_{0}(r), & 0 \leq r \leq h_{0}, \\ u(0, r)=u_{0}(r), & r \geq 0,\end{cases}
$$

where $\Delta_{r} u=u_{r r}+\frac{n-1}{r} u_{r}, \Delta_{r} v=v_{r r}+\frac{n-1}{r} v_{r}\left(r=|x|, x \in \mathbb{R}^{n}, n \geq 1\right), d, \mu$, and $h_{0}$ are given positive constants, and the functions $a, b, c, \alpha, \beta \in C^{v_{0}}([0, \infty))$ for some $v_{0} \in(0,1)$ satisfy

$$
\kappa_{1} \leq a(r), b(r), c(r), \alpha(r), \beta(r) \leq \kappa_{2}, \forall r \in[0, \infty)
$$

for given positive constants $\kappa_{1} \leq \kappa_{2}$. Here the sphere $\{r=h(t)\}$ is the moving boundary to be determined.

In this paper, we shall focus on the dynamical process of an invasive predator species with population density $v(t,|x|)$ invading into the $\mathrm{n}$-dimensional heterogeneous habitat of a native prey species with population density $u(t,|x|)$. To do so, one shall consider that the prey population is initially uniformly well disturbed and undergoes the diffusion and growth in the entire space $\mathbb{R}^{n}$, while the predator population is initially introduced in some localized location, namely, $v_{0}(|x|)$ occupies in a ball $\left\{r<h_{0}\right\}$, and disperses through random diffusion over an expanding ball $\{r<h(t)\}$, whose boundary $\{r=h(t)\}$ is the spreading front and satisfies the free boundary condition $h^{\prime}(t)=-\mu v_{r}(t, h(t))$, where $\mu$ is a given positive constant. Using such a framework, we are interested in deriving some information about the invasion of predator in the environment. Before stating our results, let us precise the assumptions on the initial data. We assume that the initial functions $u_{0}(r)$ and $v_{0}(r)$ satisfy

$$
\left\{\begin{array}{l}
u_{0} \in C^{2}([0, \infty)) \cap L^{\infty}((0,+\infty)), u_{0}>0 \text { in }[0, \infty), \\
v_{0} \in C^{2}\left(\left[0, h_{0}\right]\right), v_{0}\left(h_{0}\right)=0 \text { and } v_{0}>0 \text { in }\left(0, h_{0}\right) .
\end{array}\right.
$$

The paper is organized as follows. In Section 2, we first state the existence, boundedness and uniqueness of the solution for the problem (2), as well as some comparison principle for the following proof. Section 3 is mainly devoted to the proof of the spreading-vanishing dichotomy result. In Section 4 , some rough estimates for the spreading speed are obtained in the case that spreading of $v$ happens.

\section{Some Preliminaries}

In this section, we will give the existence, uniqueness and some estimates of the solution to the problem (2). Then, we show some comparison results, which will be used in the following sections.

Theorem 2.1. For any given $\left(u_{0}, v_{0}\right)$ satisfying (4) and any $v \in(0,1)$, there exists $T>0$ such that the problem (2) admits a unique bounded solution $(u, v, h) \in C^{\frac{1+v}{2}, 1+v}\left(D_{T}^{\infty}\right) \times$ $C^{\frac{1+v}{2}, 1+v}\left(D_{T}\right) \times C^{1+\frac{v}{2}}([0, T])$, where $D_{T}^{\infty}=\left\{(t, r) \in \mathbb{R}^{2}: t \in\right.$ $[0, T], r \in[0, \infty)\} \quad, \quad D_{T}=\left\{(t, r) \in \mathbb{R}^{2}: t \in[0, T], r \in\right.$ $[0, h(t)]\}$. Furthermore, there exist positive constants $M_{1}, M_{2}$, $M_{3}$ independent of $T$ such that

$$
\begin{gathered}
0 \leq u(t, r) \leq M_{1}, \text { for } 0<t \leq T, r \geq 0 \\
0<v(t, r) \leq M_{2}, \text { for } 0<t \leq T, 0 \leq r<h(t) \\
\left.0<h^{\prime}(t) \leq M_{3}, \text { for } 0<t \leq T .\right)
\end{gathered}
$$

Proof. Since the functions $a, b, c, \alpha$ and $\beta$ are bounds, then the proofs of the existence, uniqueness, the estimates of (5) - (6) and the first estimate of (7) can be done by modifying the arguments for a general free boundary problem in $[8,14]$. The details are omitted here.

To derive an upper bound of $h^{\prime}(t)$, we define 


$$
\Omega_{K}:=\left\{(t, r): 0<t<T, h(t)-K^{-1}<r<h(t)\right\},
$$

and construct an auxiliary function

$$
w(t, r)=M_{2}\left[2 K(h(t)-r)-K^{2}(h(t)-r)^{2}\right],
$$

where $K$ is a positive constant such that $w(t, r) \geq v(t, r)$ holds over $\Omega_{K}$. By direct calculation, for $(t, r) \in \Omega_{K}$, we can obtain

$$
\begin{aligned}
& w_{t}=2 M_{2} K h^{\prime}(t)[1-K(h(t)-r)] \geq 0, \\
& -w_{r}=2 M_{2} K[1-K(h(t)-r)] \geq 0, \\
& -w_{r r}=2 M_{2} K^{2}, v\left(\alpha(r)-\frac{\beta(r) v}{u+\delta}\right) \leq M_{2} \kappa_{2} .
\end{aligned}
$$

Then, we can choose $K$ satisfying $K^{2} \geq \frac{\kappa_{2}}{2}$ such that

$$
v_{r}(t, h(t)) \geq w_{r}(t, h(t))=-2 M_{2} K, h^{\prime}(t
$$

This completes the proof.

Theorem 2.2. If the conditions in Theorem 2.1 still hold, then the solution of the problem (2) defined in Theorem 2.1 can be extended uniquely to all $t \in(0, \infty)$.

Proof. Set $\left[0, T_{\max }\right)$ be the maximal time interval in which the solution exists. It is easy to show $T_{\max }>0$ through Theorem 2.1. We only need to prove $T_{\max }=\infty$. On the contrary, we assume $T_{\max }<\infty$. Due to Theorem 2.1, the standard $L^{p}$ estimates and the Sobolev embedding theorem, we can find a constant $C>0$ depending only on $M_{i}(i=$ $1,2,3)$ such that $u$ is continuous for $(t, r) \in\left[0, T_{\text {max }}\right) \times$ $[0, \infty)$ and $\|v(t, \cdot)\|_{C^{1+\frac{\alpha}{2}}([0, h(t)])} \leq C$. Then from the proof of

$$
w_{t}-\left(w_{r r}+\frac{n-1}{r} w_{r}\right) \geq 2 M_{2} K^{2} \geq v\left(\alpha(r)-\frac{\beta(r) v}{u+\delta}\right) \text { in } \Omega_{K} .
$$

Moreover, we have $w\left(t, h(t)-K^{-1}\right)=M_{2} \geq v(t, h(t)-$ $\left.K^{-1}\right)$ and $w(t, h(t))=v(t, h(t))=0$. Since $v_{0}(r)=$ $-\int_{r}^{h_{0}} v^{\prime}{ }_{0}(s) d s \leq\left(h_{0}-r\right)\left\|v^{\prime}{ }_{0}\right\|_{C\left(\left[0, h_{0}\right]\right)}$ in $\left[h_{0}-K^{-1}, h_{0}\right]$, and $\quad w(0, r)=M_{2}\left[2 K\left(h_{0}-r\right)-K^{2}\left(h_{0}-r\right)^{2}\right] \geq$ $M_{2} K\left(h_{0}-r\right)$ in $\left[h_{0}-K^{-1}, h_{0}\right]$, it is easy to show that if $K M_{2} \geq\left\|v_{0}^{\prime}\right\|_{C\left(\left[0, h_{0}\right]\right)}$, then

$v_{0}(r) \leq\left(h_{0}-r\right)\left\|v^{\prime}{ }_{0}\right\|_{C\left(\left[0, h_{0}\right]\right)} \leq w(0, r)$ in $\left[h_{0}-K^{-1}, h_{0}\right]$.

Set $K=\max \left\{\sqrt{\frac{\kappa_{2}}{2}}, \frac{\left\|v_{0}\right\|_{C\left(\left[0, h_{0}\right]\right)}}{M_{2}}\right\}$, by the maximum principle to $w-v$ over $\Omega_{K}$, we can obtain that $v(t, r) \leq w(t, r)$ for $(t, r) \in \Omega_{K}$, which implies that

$h^{\prime}(t)=-\mu v_{r}(t, h(t)) \leq 2 \mu M_{2} K=: M_{3}$.

Theorem 2.1, there exists $\tau>0$ depending only on $C$ and $M_{i}(i=1,2,3)$ such that the solution of problem (2) with initial time $T_{\max }-\frac{\tau}{2}$ can be extended uniquely to the time $T_{\max }-\frac{\tau}{2}+\tau$. It is contradicted with the assumption. The proof is now completed.

Following we present some comparison principles which will be used in the following sections.

Lemma 2.1. (Comparison Principle). Assume that $T \in$ $(0, \infty), \bar{h} \in C^{1}([0, T]), \quad \bar{u} \in L^{\infty}\left(D_{T}^{\infty}\right) \cap C^{1,2}\left(D_{T}^{\infty}\right), \quad \bar{v} \in$ $C\left(\bar{G}_{T}^{*}\right) \cap C^{1,2}\left(G_{T}^{*}\right) \quad$ with $\quad G_{T}^{*}=\left\{(t, r) \in \mathbb{R}^{2}: t \in(0, T], r \in\right.$ $(0, \bar{h}(t))\}$. If $(\bar{u}, \bar{v}, \bar{h})$ satisfies

$$
\begin{cases}\bar{u}_{t}-d \Delta_{r} \bar{u} \geq \bar{u}(a(r)-b(r) \bar{u}), & t>0, r>0, \\ \bar{v}_{t}-\Delta_{r} \bar{v} \geq \bar{v}\left(\alpha(r)-\frac{\beta(r) \bar{v}}{\bar{u}+\delta}\right), & t>0,0<r<\bar{h}(t), \\ \bar{u}_{r}(t, 0)=0, \bar{v}_{r}(t, 0)=0, & t>0, \\ \bar{v}(t, \bar{h}(t))=0, \bar{h}^{\prime}(t) \geq-\mu \bar{v}_{r}(t, \bar{h}(t)), & t>0,\end{cases}
$$

and

$$
\begin{gathered}
\bar{h}(0) \geq h_{0}, \bar{v}(0, r) \geq 0 \text { in }[0, \bar{h}(0)], \\
u_{0}(r) \leq \bar{u}(0, r) \text { in }[0, \infty), v_{0}(r) \leq \bar{v}(0, r) \text { in }\left[0, h_{0}\right],
\end{gathered}
$$

then the solution $(u, v, h)$ of the equation (2) satisfies

$$
u(t, r) \leq \bar{u}(t, r) \text { on } D_{\underline{T}}^{\infty}, v(t, r) \leq \bar{v}(t, r) \text { on } D_{T}, h(t) \leq
$$$$
\bar{h}(t) \text { on }[0, T] \text {, }
$$

where $D_{T}^{\infty}$ and $D_{T}$ are defined in Theorem 2.1.

Proof. For the case $h_{0}<\bar{h}(0)$, we claim that $h(t)<\bar{h}(t)$ for all $t \in(0, T]$. If our claim does not hold, then there exists a first $t^{*} \leq T$ such that $h(t)<\bar{h}(t)$ for $t \in\left(0, t^{*}\right)$ and $h\left(t^{*}\right)=\bar{h}\left(t^{*}\right)$. It is easy to see

$$
h^{\prime}\left(t^{*}\right) \geq \bar{h}^{\prime}\left(t^{*}\right)
$$

We first show that $u \leq \bar{u}$ in $\left[0, t^{*}\right] \times[0, \infty)$. Setting $U=\bar{u}-u$, we get that $U$ satisfies

$$
\left\{\begin{array}{l}
U_{t}-d \Delta_{r} U \geq\left(\kappa_{1}-2 \kappa_{2} \widetilde{M_{1}}\right) U, 0<t \leq t^{*}, r>0 \\
U_{r}(t, 0)=0,0<t \leq t^{*} \\
U(0, r) \geq 0, r \geq 0
\end{array}\right.
$$

where $\widetilde{M_{1}}$ is an upper bound of $\bar{u}$ and $u$ in $[0, T] \times[0, \infty)$. By the maximum principle, we can obtain that $U(t, r) \geq 0$ in $\left[0, t^{*}\right] \times[0, \infty)$, that is $u \leq \bar{u}$ in $\left[0, t^{*}\right] \times[0, \infty)$.

Letting $W=(\bar{v}-v) e^{-k t}$, it is easy to show that $W$ satisfies

$$
\left\{\begin{array}{l}
W_{t}-\Delta_{r} W \geq\left(\kappa_{1}-k-2 \kappa_{2} \delta^{2} \widetilde{M_{1}} \widetilde{M_{2}}\right) W, 0<t \leq t^{*}, 0<r<h(t) \\
W(t, r)=0,0<t \leq t^{*}, r \geq \bar{h}(t) \\
W_{r}(t, 0)=0,0<t \leq t^{*} \\
W(0, r) \geq 0, r \geq 0
\end{array}\right.
$$

where $\widetilde{M_{2}}$ is an upper bound of $\bar{v}$ and $v$ in $[0, T] \times[0, \infty)$ and

$k$ is sufficiently large such that $k \geq 2 \kappa_{2} \delta^{2} \widetilde{M_{1}} \widetilde{M_{2}}+1+\kappa_{1}$. 
Since the first inequality of (10) holds only in part of $[0, \infty)$, the maximum principle cannot be used directly. We can prove that for any $l>h\left(t^{*}\right)$,

$$
W(t, r) \geq-\frac{\widetilde{M_{2}}\left(r^{2}+2 n t\right)}{l^{2}} \text { in }\left[0, t^{*}\right] \times[0, l]
$$

$$
\left\{\begin{array}{l}
\bar{W}_{t}-\Delta_{r} \bar{W} \geq\left(\kappa_{1}-k-2 \kappa_{2} \delta^{2} \widetilde{M_{1}} \widetilde{M_{2}}\right) \bar{W}, 0<t \leq t^{*}, 0<r<h(t), \\
\bar{W}(t, r) \geq \frac{\widetilde{M}_{2}\left(r^{2}+2 n t\right)}{l^{2}}>0,0<t \leq t^{*}, h(t) \leq r \leq l, \\
\bar{W}_{r}(t, 0)=0,0<t \leq t^{*}, \\
\bar{W}(0, r) \geq 0,0 \leq r \leq l .
\end{array}\right.
$$

We need only to prove $\min _{\left[0, t^{*}\right] \times[0, l]} \bar{W}:=\gamma \geq 0$. In fact, if $\gamma<0$, then there exists $\left(t_{1}, r_{1}\right) \in \mathbb{R}^{2}$ with $0<t_{1} \leq t^{*}$ and $0 \leq r_{1}<h\left(t_{1}\right)$ such that $\bar{W}\left(t_{1}, r_{1}\right)=\gamma<0$. It is easy to show $\left(\bar{W}_{t}-\Delta_{r} \bar{W}\right)\left(t_{1}, r_{1}\right) \leq 0$. But due to our choice of $k$, we have

$$
\left(\kappa_{1}-k-2 \kappa_{2} \delta^{2} \widetilde{M_{1}} \widetilde{M_{2}}\right) \bar{W}\left(t_{1}, r_{1}\right)=\left(\kappa_{1}-k-2 \kappa_{2} \delta^{2} \widetilde{M_{1}} \widetilde{M_{2}}\right) \gamma \geq-\gamma>0 .
$$

It is a contradiction. Then, it is easy to get that $\bar{W} \geq 0$ in $\left[0, t^{*}\right] \times[0, l]$, which implies that

$$
W(t, r) \geq-\frac{\widetilde{M_{2}}\left(r^{2}+2 n t\right)}{l^{2}} \text { for }\left[0, t^{*}\right] \times[0, l] .
$$

Since $V(t, r)=\bar{v}(t, r)-v(t, r)$ satisfies

$V_{t}-\Delta_{r} V \geq\left(\kappa_{1}-2 \kappa_{2} \delta^{2} \widetilde{M_{1}} \widetilde{M_{2}}\right) V, 0<t \leq t^{*}, 0<r<h(t)$,

we can use the strong maximum principle and the Hopf boundary lemma to obtain that $V(t, r)>0$ in $\left(0, t^{*}\right] \times$ $[0, h(t)]$, and $V_{r}\left(t^{*}, h\left(t^{*}\right)\right)<0$. Then we deduce $h^{\prime}\left(t^{*}\right)<$ $\bar{h}^{\prime}\left(t^{*}\right)$. This contradicts with (9). This proves our claim that $h(t)<\bar{h}(t)$ for all $t \in(0, T]$.

Apply the above produce over $[0, T] \times[0, \infty)$, we can conclude that $u \leq \bar{u}$ and $v \leq \bar{v}$ in $[0, T] \times[0, \infty)$. Moreover, $v \leq \bar{v}$ in $[0, T] \times[0, h(t))$. If $h_{0}=\bar{h}(0)$, let $\left(u_{\epsilon}, v_{\epsilon}, h_{\epsilon}\right)$ denote the unique solution of (2) with $h_{0}$ replaced by $h_{0}(1-\epsilon)$ for small $\epsilon>0$. From the continuous dependence on parameter of the solution to (2), it is easy to show that $\left(u_{\epsilon}, v_{\epsilon}, h_{\epsilon}\right)$ converges to $(u, v, h)$. The desired results are followed by letting $\epsilon \rightarrow 0$ in the inequalities $u_{\epsilon} \leq \bar{u}, v_{\epsilon} \leq \bar{v}$ and $h_{\epsilon} \leq \bar{h}$.

Lemma 2.2. (Comparison Principle). Let $T \in(0, \infty)$, $\underline{h} \in C^{1}([0, T])$ with $\underline{h}>0$ for all $t \in[0, T]$, and $\underline{v} \in$ $\bar{C}\left(\bar{G}_{T}^{* *}\right) \cap C^{1,2}\left(G_{T}^{* *}\right)$ with $G_{T}^{* *}=\left\{(t, r) \in \mathbb{R}^{2}: t \in(0, T], \bar{r} \in\right.$ $(0, \underline{h}(t))\}$. Suppose that $(\underline{v}, \underline{h})$ satisfies

$$
\begin{cases}\underline{v}_{t}-\Delta_{r} \underline{v} \leq \underline{v}\left(\alpha(r)-\beta(r) \delta^{-1} \underline{v}\right), & t>0,0<r<\underline{h}(t), \\ \underline{v}_{r}(t, 0)=0, \underline{v}(t, \underline{h}(t))=0, & t>0, \\ \underline{h}^{\prime}(t) \leq-\mu \underline{v}_{r}(t, \underline{h}(t)), & t>0,\end{cases}
$$

and

$$
\underline{h}(0) \leq h_{0} \text {, and } v_{0}(r) \geq \underline{v}(0, r) \text { in }[0, \underline{h}(0)],
$$

then the solution $(u, v, h)$ of the equation (2) satisfies

$$
v(t, r) \geq \underline{v}(t, r) \text { in } \bar{G}_{T}^{* *}, h(t) \geq \underline{h}(t) \text { in }[0, T] .
$$

We omit the details of the proof which can be proved as the process with the above lemma.

\section{The Spreading-Vanishing Dichotomy}

In this section, we prove the spreading-vanishing dichotomy of the free boundary problem (2). By the estimate of $h^{\prime}(t)$ in Theorem 2.1, it is easy to show that there exists $h_{\infty} \in(0, \infty]$ such that $\lim _{t \rightarrow \infty} h(t)=h_{\infty}$.

Let $\lambda_{1}(d, \alpha, R)$ be the principal eigenvalue for the following problem

$$
\begin{cases}-d \Delta \varphi=\lambda \alpha(|x|) \varphi, & x \in B_{R} \\ \varphi=0, & x \in \partial B_{R}\end{cases}
$$

where $\alpha(r)>\frac{\kappa_{1}}{2}$ for $r \in[0, \infty), \alpha(r) \in C^{1}([0, \infty))$ and $B_{R}$ stands for the ball with center at 0 and radius $R$. We can know in that $\lambda_{1}(d, \alpha, R)$ is a strictly decreasing continuous function in $R$ and satisfies

$$
\lim _{R \rightarrow 0^{+}} \lambda_{1}(d, \alpha, R)=\infty \text { and } \lim _{R \rightarrow \infty} \lambda_{1}(d, \alpha, R)=0 .
$$

Therefore, for fixed $d>0$ and $\alpha \in C^{1}([0, \infty))$, there exists a unique $R^{*}(d, \alpha)$ such that $\lambda_{1}\left(d, \alpha, R^{*}(d, \alpha)\right)=1$, $\lambda_{1}(d, \alpha, R)<1$ for $R>R^{*}(d, \alpha)$, and $\lambda_{1}(d, \alpha, R)>1$ for $R<R^{*}(d, \alpha)$. Since $\lambda_{1}(d, \alpha, R)$ is a strictly decreasing continuous function in $\alpha$ and $R$, we have that $R^{*}(d, \alpha)$ is a strictly decreasing continuous function in $\alpha$.

In order to investigate asymptotic properties of solutions for the problem (2), we first recall the following spreadingvanishing dichotomy for the radially symmetric diffusive logistic problem

$$
\begin{cases}w_{t}-d \Delta_{r} w=w(\alpha(r)-\beta(r) w), & t>0,0<r<h(t) \\ w_{r}(t, 0)=0, w(t, h(t))=0, & t>0 \\ h^{\prime}(t)=-\mu w_{r}(t, h(t)), & t>0 \\ h(0)=h_{0}, w(0, r)=w_{0}(r), & 0 \leq r \leq h_{0} .\end{cases}
$$

Theorem 3.1. (spreading-vanishing dichotomy)(See [Du and Guo [8], Theorem 2.4]) Let $(w(t, r), h(t))$ be the solution of the free boundary problem (11). Then one of the following holds:

(i) spreading: $\quad h_{\infty}=\infty$ and $\lim _{t \rightarrow \infty} w(t, r)=\widehat{w}(r)$ uniformly in any compact subset of $[0, \infty)$, where $\widehat{w}(|x|)$ is the unique positive (radial) solution of the equation

$$
-d \Delta \widehat{w}=\widehat{w}(\alpha(|x|)-\beta(|x|) \widehat{w}) \text { in } \mathbb{R}^{n} ;
$$


(ii) vanishing: $\quad h_{\infty} \leq R^{*}(d, \alpha)$ and $\lim _{t \rightarrow \infty} \| w(t$, ) $\|_{C([0, h(t)])}=0$.

Theorem 3.2. (See [Du and Guo [8]]) If $h_{0} \geq R^{*}(d, \alpha)$, then spreading always happens. If $h_{0}<R^{*}(d, \alpha)$, then there exists $\mu^{*}>0$ depending on $w_{0}$ such that vanishing happens when $\mu \leq \mu^{*}$ and spreading happens when $\mu>\mu^{*}$.

We will give the following lemmas to obtain the asymptotic properties of solutions for the problem (2).

Lemma 3.1. If $h_{\infty}<\infty$, then

$$
\lim _{t \rightarrow \infty} u(t, r)=U(r) \text { uniformly in any compact subset of }[0, \infty) \text {, }
$$

where $U(|x|)$ is the unique positive (radial) solution of the equation

$$
-d \Delta u=u(a(|x|)-b(|x|) u), x \in \mathbb{R}^{n},
$$

and

$$
\lim _{t \rightarrow \infty}\|v(t, \cdot)\|_{C([0, h(t)])}=0 .
$$

Proof. By the similar way to that of [34, Theorem 2.1], we can get some uniform estimates of $\left(u, v, h^{\prime}\right)$. Then according to Lemma of [36], we can obtain that (14) holds. The details are omitted here.

We use a squeezing argument introduced in [16] to state that (12) holds. Considering the Dirichlet problem

$$
-d \Delta_{r} z=z(a(r)(1-\varepsilon)-b(r) z), r<R, z(R)=0,
$$

where $\varepsilon>0$ is a given constant, and the boundary blow-up problem

$$
\begin{cases}z_{t}-d \Delta_{r} z=z\left(a(r)\left(1-\varepsilon_{i}\right)-b(r) z\right), & t>T_{i}, 0<r<R_{i} \\ z_{r}(t, 0)=0, z\left(t, R_{i}\right)=0, & t>T_{i} \\ z\left(T_{i}, r\right)=u\left(T_{i}, r\right), & 0 \leq r \leq R_{i} .\end{cases}
$$

Since $\lambda_{1}\left(d, a(r)\left(1-\varepsilon_{i}\right), R_{i}\right)<1$, it is well known (see, Proposition 3.3 in [3]) that (15) admits a unique positive solution $z_{i}(t, r)$. Moreover,

$$
\begin{array}{r}
\lim _{t \rightarrow \infty} z_{i}(t, r)=z_{R_{i}}^{\varepsilon_{i}}(r) \text { uniformly for } r \in\left[0, R_{i}\right] . \\
\liminf _{t \rightarrow \infty} u(t, r) \geq U(r) \text { uniformly in any compact subset of }[0, \infty) .
\end{array}
$$

Similarly, by arguments to these in the proof of Theorem 4.1 of [16], it is easy to show that

$$
\underset{t \rightarrow \infty}{\limsup } u(t, r) \leq w_{R_{i}}(r) \text { uniformly for } r \in\left[0, R_{i}\right]
$$

which implies that

$$
\underset{t \rightarrow \infty}{\limsup } u(t, r) \leq U(r) \text { uniformly in any compact subset of }[0, \infty) \text {. }
$$

From (16) and (17), we can obtain that (12) is true.

Lemma 3.2. If $h_{\infty}<\infty$, then $h_{\infty} \leq R^{*}(1, \alpha)$.

Proof. On the contrary, we assume $h_{\infty}>R^{*}(1, \alpha)$. Since $R^{*}(1, \alpha)$ is a strictly decreasing continuous function in $\alpha$, then for any given $\varepsilon_{1}>0$ sufficiently small, there exists $\tau \gg 1$ such that $h(\tau)>\max \left\{h_{0}, R^{*}\left(1, \alpha-\varepsilon_{1}\right)\right\}$.

We can take advantage of the comparison principle to show that $v(t, r) \geq w(t, r), \forall t \geq \tau, 0 \leq r \leq h(\tau)$, where $w=w(t, r)$ is the positive solution of the following initial boundary value problem with fixed boundary

$$
\begin{cases}w_{t}=\Delta_{r} w+w\left(\alpha(r)-\varepsilon_{1}-\beta(r) \delta^{-1} w\right), & t>\tau, 0<r<h(\tau), \\ w_{r}(t, 0)=0, w(t, h(\tau))=0, & t>\tau, \\ w(\tau, r)=v(\tau, r), & 0 \leq r \leq h(\tau) .\end{cases}
$$

Since $\lambda_{1}\left(1, \alpha-\varepsilon_{1}, h(\tau)\right)<\lambda_{1}\left(1, \alpha-\varepsilon_{1}, R^{*}\left(1, \alpha-\varepsilon_{1}\right)\right)=1$, we can know from [3] that $w(t, r) \rightarrow w^{*}(r)$ as $t \rightarrow \infty$ 
uniformly for $r \in[0, h(\tau)]$, where $w^{*}$ is the unique positive solution of

$$
\left\{\begin{array}{l}
\Delta_{r} w^{*}+w^{*}\left(\alpha(r)-\varepsilon_{1}-\beta(r) \delta^{-1} w^{*}\right)=0, r \in(0, h(\tau)) \\
w_{r}^{*}(0)=w^{*}(h(\tau))=0
\end{array}\right.
$$

Hence

$\liminf _{t \rightarrow \infty} v(t, r) \geq \lim _{t \rightarrow \infty} w(t, r)=w^{*}(r)>0$ in $[0, h(\tau))$.

This is a contradiction with (14). Therefore, $h_{\infty} \leq$ $R^{*}(1, \alpha)$ holds.

Lemma 3.3. Let $(u, v, h)$ be any solution of the equation (2). If $h_{\infty}=\infty$, then

$$
V_{1}(r) \leq \liminf _{t \rightarrow \infty} v(t, r) \leq \limsup _{t \rightarrow \infty} v(t, r) \leq V_{2}(r)
$$

uniformly in any compact subset of $[0, \infty)$, where $V_{1}(|x|)$ and $V_{2}(|x|)$ are unique positive (radial) solutions of the equations

$$
-\Delta v=v\left(\alpha(|x|)-\beta(|x|) \delta^{-1} v\right), x \in \mathbb{R}^{n},
$$

and

$$
-\Delta v=v\left(\alpha(|x|)-\beta(|x|) U^{-1}(|x|) v\right), x \in \mathbb{R}^{n},
$$

respectively, where $U(|x|)$ is defined in Lemma 3.1.

Proof. Since $h_{\infty}=\infty$, there exists $T>0$ such that $h(T)>R^{*}(1, \alpha)$. We choose a function $\underline{v}_{0}(r)$ satisfying $\underline{v}_{0} \in C^{2}([0, h(t)]), \underline{v}_{0}(r) \leq v(T, r)$ in $[0, h(T)], \underline{v}_{0}(r)>0$ in $(0, h(T))$ and $\underline{v}_{0}(h(T))=0$ and consider the following problem

$$
\begin{cases}\underline{v}_{t}-\Delta_{r} \underline{v}=\underline{v}\left(\alpha(r)-\beta(r) \delta^{-1} \underline{v}\right), & t>T, 0<r<\underline{h}(t), \\ \underline{v}_{r}(t, 0)=\underline{v}(t, \underline{h}(t))=0, & t>T, \\ \underline{h}(t)=-\mu \underline{v}_{r}(t, \underline{h}(t)), & t>T, \\ \underline{h}(T)=h(T), \underline{v}(T, r)=\underline{v}_{0}(r), & 0 \leq r \leq h(T) .\end{cases}
$$

By Theorem 2.1 of [8], this problem has a unique solution $(\underline{v}, \underline{h})$ for all $t>T$. In view of Lemma 2.2, we have

$$
\begin{aligned}
& v(t, r) \geq \underline{v}(t, r) \text { for } t \geq T, 0 \leq r \leq \underline{h}(t), \\
& h(t) \geq \underline{h}(t) \text { for } t \geq T .
\end{aligned}
$$

Using Theorem 3.1, we can see that

$$
\lim _{t \rightarrow \infty} v(t, r)=V_{1}(r) \text { uniformly in any compact subset of }[0, \infty) .
$$

It follows from (18) and (19) that

$$
\liminf _{t \rightarrow \infty} v(t, r) \geq V_{1}(r) \text { uniformly in any compact subset of }[0, \infty) .
$$

We define

$$
\bar{u}(t, r)=\left(1+\frac{\kappa_{2}\left\|u_{0}\right\|_{L^{\infty}}([0, \infty))}{\kappa_{1}} e^{-\left(\kappa_{1}^{2} / \kappa_{2}\right) t}\right) U(r) .
$$

Since $U(r)$ satisfies $(13)$ and $\kappa_{1} \leq a(r), b(r) \leq \kappa_{2}$, it is easy to show that $\frac{\kappa_{1}}{\kappa_{2}} \leq U(r) \leq \frac{\kappa_{2}}{\kappa_{1}}$ for $r \in[0, \infty)$. By direct calculation, we can obtain

$$
\begin{aligned}
& \bar{u}_{t}-d \Delta_{r} \bar{u}-\bar{u}(a(r)-b(r) \bar{u}) \\
= & \frac{\kappa_{2}\left\|u_{0}\right\|_{L^{\infty}([0, \infty))}}{\kappa_{1}} e^{-\left(\kappa_{1}^{2} / \kappa_{2}\right) t} U(r)\left[-\frac{\kappa_{1}^{2}}{\kappa_{2}}+\left(1+\frac{\kappa_{2}\left\|u_{0}\right\|_{L^{\infty}}([0, \infty))}{\kappa_{1}} e^{-\left(\kappa_{1}^{2} / \kappa_{2}\right) t}\right) b(r) U(r)\right] \\
\geq & 0,
\end{aligned}
$$

and $\bar{u}(0, r)=\left(1+\frac{\kappa_{2}\left\|u_{0}\right\|_{L^{\infty}([0, \infty))}}{\kappa_{1}}\right) U(r)>\left\|u_{0}\right\|_{L^{\infty}([0, \infty))} \geq u_{0}(r)$. Due to $\lim _{t \rightarrow \infty} \bar{u}(t, r)=U(r)$ uniformly in $[0, \infty)$, for any given $\epsilon>0$, there exists $T_{\epsilon}>T$ such that $\bar{u}(t, r) \leq U(r)(1+\epsilon), \forall t \geq T_{\epsilon}, r \in[0, \infty)$. Then we consider the auxiliary problem

$$
\begin{cases}\bar{v}_{t}-\Delta_{r} \bar{v}=\bar{v}\left(\alpha(r)-\frac{\beta(r)}{U(r)(1+\epsilon)+\delta} \bar{v}\right), & t>T_{\epsilon}, 0<r<\bar{h}(t), \\ \bar{v}_{r}(t, 0)=0, \bar{v}(t, \bar{h}(t))=0, & t>T_{\epsilon}, \\ \bar{h}^{\prime}(t)=-\mu \bar{v}_{r}(t, \bar{h}(t)), & t>T_{\epsilon}, \\ \bar{h}\left(T_{\epsilon}\right)=h\left(T_{\epsilon}\right), \bar{v}\left(T_{\epsilon}, r\right)=v\left(T_{\epsilon}, r\right), & 0 \leq r \leq \bar{h}\left(T_{\epsilon}\right),\end{cases}
$$

and deduce that $(U(1+\epsilon), \bar{v}, \bar{h})$ satisfies (8) for $t>T_{\epsilon}$. Though Lemma 2.1, it is easy to show that

$$
\bar{h}(t) \geq h(t), \bar{v}(t, r) \geq v(t, r), \forall t>T_{\epsilon}, 0<r<h(t) .
$$

Hence, $\bar{h}(\infty)=\infty$. By Theorem 3.1, it is easy to show that

$$
\lim _{t \rightarrow \infty} \bar{v}(t, r) \leq V^{\epsilon}(r) \text { uniformly in any compact subset of }[0, \infty),
$$

where $V^{\epsilon}(|x|)$ is the unique radial positive solution of the following equation 


$$
-\Delta v=v\left[\alpha(|x|)-\frac{\beta(|x|)}{U(|x|)(1+\epsilon)+\delta} v\right], x \in \mathbb{R}^{n} .
$$

Since $V^{\epsilon}(|x|)$ is continuously dependent on $\epsilon$, letting $\epsilon \rightarrow 0$, we can obtain that

$$
\limsup v(t, r) \leq V_{2}(r)
$$

uniformly in any compact subset of $[0, \infty)$.

Here completes the proof.

By the above Lemmas 3.1 3.3, it is easy to show the following dichotomy result.

Theorem 3.3. Suppose that $(u, v, h)$ is the unique solution of the equation (2) with the initial condition (4). Then the following alternative holds.

Either

(i) spreading of $v: h_{\infty}=\infty$ and
$V_{1}(r) \leq \liminf _{t \rightarrow \infty} v(t, r) \leq \limsup _{t \rightarrow \infty} v(t, r) \leq V_{2}(r)$ uniformly in any compact subset of $[0, \infty)$;

or

(ii) vanishing of $v: h_{\infty} \leq R^{*}(1, \alpha)$ and $\lim _{t \rightarrow \infty} \| v(t$, ) $\|_{C([0, h(t)])}=0$.

Theorem 3.4. In Theorem 3.3, if $h_{0} \geq R^{*}(1, \alpha)$, then spreading of $v$ always happens. If $h_{0}<R^{*}(1, \alpha)$, then there exist $\mu^{*} \geq \mu_{*}>0$ depending on $\left(u_{0}, v_{0}\right)$ such that the spreading of $v$ happens exactly when $\mu>\mu^{*}$ and vanishing of $v$ occurs exactly when $\mu \leq \mu_{*}$.

For the case $h_{0} \geq R^{*}(1, \alpha)$, since that $h^{\prime}(t)>0$ for $t>0$ which is obtained in Theorem 2.1, we have $h_{\infty}>R^{*}(1, \alpha)$. Hence Lemmas 3.2 and 3.3 imply the spreading result. We prove the result for the case $h_{0}<R^{*}(1, \alpha)$ by the following lemmas.

Lemma 3.4. If $h_{0}<R^{*}(1, \alpha)$, then there exists $\underline{\mu}>0$ depending on $\left(u_{0}, v_{0}\right)$ such that $h_{\infty}=\infty$ when $\mu \geq \underline{\mu}$.

Proof. Considering the following auxiliary problem

$$
\begin{cases}\underline{v}-\Delta_{r} \underline{v}=\underline{v}\left(\alpha(r)-\beta(r) \delta^{-1} \underline{v}\right), & t>0,0<r<\underline{h}(t), \\ \underline{v}_{r}(t, 0)=0, \underline{v}(t, \underline{h}(t))=0, & t>0, \\ \underline{h}^{\prime}(t)=-\mu \underline{v}_{r}(t, \underline{h}(t)), & t>0, \\ \underline{h}(0)=h_{0}, \underline{v}(0, r)=v_{0}(r), & 0 \leq r \leq h_{0} .\end{cases}
$$

By Lemma 2.2, we have that

$$
\underline{h}(t) \leq h(t), \underline{v}(t, r) \leq v(t, r), \forall t>0,0<r<\underline{h}(t) .
$$

Since $\underline{h}(0)=h_{0}<R^{*}(1, \alpha)$, by Lemma 2.8 of [8], there exists $\mu>0$ such that $\underline{h}_{\infty}=\infty$ for $\mu \geq \underline{\mu}$. Therefore, $h_{\infty}=\infty$ for $\mu \geq \mu$. The proof is finished.

Lemma 3.5. Suppose $h_{0}<R^{*}(1, \alpha)$. Then there exists $\bar{\mu}>0$ depending on $v_{0}$ such that $h_{\infty}<+\infty$ if $\mu \leq \bar{\mu}$.

Proof. Using Lemma 2.1, it is easy to show that $\left(\frac{\kappa_{2}}{\kappa_{1}}, \bar{v}(t, r), \bar{h}(t)\right)$ is an upper solution for (2), where $(\bar{v}(t, r), \bar{h}(t))$ is a solution of the following problem

$$
\begin{cases}\bar{v}_{t}-\Delta_{r} \bar{v}=\bar{v}\left(\alpha(r)-\beta(r) \frac{\bar{v}}{\frac{\kappa_{2}}{\kappa_{1}}+\delta}\right), & t>0,0<r<\bar{h}(t), \\ \bar{v}_{r}(t, 0)=0, \bar{v}(t, \bar{h}(t))=0, & t>0, \\ \bar{h}^{\prime}(t)=-\mu \bar{v}_{r}(t, \bar{h}(t)), & t>0, \\ \bar{h}(0)=h_{0}, \bar{v}(0, r)=v_{0}(r), & 0 \leq r \leq h_{0} .\end{cases}
$$

Since $\bar{h}(0)=h_{0}<R^{*}(1, \alpha)$, it follows from Theorem 3.2 that there exists $\bar{\mu}>0$ depending on $v_{0}$ such that $\bar{h}_{\infty}<\infty$ if $\mu \leq \bar{\mu}$. Therefore, $h_{\infty}<\infty$ for $\mu \leq \bar{\mu}$. The proof is completed.

Lemma 3.6. Suppose $h_{0}<R^{*}(1, \alpha)$. Then there exist $\mu^{*} \geq \mu_{*}>0$ depending on $\left(u_{0}, v_{0}\right)$ such that $h_{\infty} \leq R^{*}(1, \alpha)$ if $\mu \leq \mu_{*}$ and $h_{\infty}=\infty$ if $\mu>\mu^{*}$.

Proof. The proof can be found in many papers, such as Theorem 3.5 of [38], Theorem 4.11 of [24], et al. For the convenience of the readers, we give the outline of the proof of this result.

In order to clarify the dependence of the solution for (2) on $\mu$, we will write $\left(u_{\mu}, v_{\mu}, h_{\mu}\right)$ in place of $(u, v, h)$. Define
$\Sigma^{*}=\left\{\mu>0: h_{\mu, \infty} \leq R^{*}(1, \alpha)\right\}$. By Lemmas 3.5 and 3.2, $(0, \bar{\mu}] \subset \Sigma^{*}$. In view of Lemma 3.4, $\Sigma^{*} \cap[\mu, \infty)=\emptyset$. Therefore, set $\mu^{*}:=\sup \Sigma^{*} \in[\bar{\mu}, \mu]$. By the definition of $\Sigma^{*}$ and Lemma 3.2, we can deduce that $h_{\mu, \infty}=\infty$ when $\mu>\mu^{*}$. We will show that $\mu^{*} \in \Sigma^{*}$. Otherwise, it is easy to get that $h_{\mu^{*}, \infty}=\infty$ and there exists $T>0$ such that $h_{\mu^{*}}(T)>$ $R^{*}(1, \alpha)$. By the continuous dependence on parameter $\mu$ of $\left(u_{\mu}, v_{\mu}, h_{\mu}\right)$, there exists a constant $\epsilon>0$ such that $h_{\mu}(T)>$ $R^{*}(1, \alpha)$ for $\mu \in\left[\mu^{*}-\epsilon, \mu^{*}+\epsilon\right]$. It follows that for all such $\mu, \lim _{t \rightarrow \infty} h_{\mu}(t)>h_{\mu}(T)>R^{*}(1, \alpha)$. This implies that $\left[\mu^{*}-\epsilon, \mu^{*}+\epsilon\right] \cap \Sigma^{*}=\varnothing \quad$ and $\sup \Sigma^{*} \leq \mu^{*}-\epsilon \quad$. This contradicts with the definition of $\mu^{*}$.

Set $\Sigma_{*}=\left\{v>0: v \geq \bar{\mu}\right.$ such that $h_{\mu, \infty} \leq R^{*}(1, \alpha)$ for all $\mu \leq v\}$, where $\bar{\mu}$ is given in Lemma 3.5. Then $\mu_{*}:=\sup \Sigma_{*} \leq$ $\mu^{*}$ and $\left(0, \mu_{*}\right) \subset \Sigma_{*}$. Similar to the above argument, it is easily obtained $\mu_{*} \in \Sigma_{*}$. The proof is completed.

\section{Estimates of the Spreading Speed}

When spreading of $v$ happens, we give some rough estimates on the spreading speed of $h(t)$ in this section.

Proposition 4.1 (See [Du and Lin [13], Proposition 4.1]) For any given constants $a>0, b>0, d>0$ and $k \in$ $[0,2 \sqrt{a d})$, the problem

$$
-d U^{\prime \prime}+k U^{\prime}=a U-b U^{2} \text { in }(0, \infty), U(0)=0,
$$

admits a unique positive solution $U=U_{k}=U_{a, b, k}$, and it satisfies $U(r) \rightarrow \frac{a}{b}$ as $r \rightarrow \infty$. Moreover, $U^{\prime}{ }_{k}(r)>0$ for $r \geq 0$, 
$U^{\prime}{ }_{k_{1}}(0)>U^{\prime}{ }_{k_{2}}(0), U_{k_{1}}(r)>U_{k_{2}}(r)$ for $r>0$ and $k_{1}>k_{2}$, and for each $\mu>0$, there exists a unique $k_{0}=k_{0}(\mu, a, b) \in$ $(0, \sqrt{2 a d})$ such that $\mu U_{k_{0}}^{\prime}(0)=k_{0}$. Furthermore,

$$
\lim _{\frac{a \mu}{b d} \rightarrow \infty} \frac{k_{0}}{\sqrt{a d}}=2, \lim _{\frac{a \mu}{b d} \rightarrow 0} \frac{k_{0}}{\sqrt{a d}} \frac{b d}{a \mu}=\frac{1}{\sqrt{3}} .
$$

It was shown in [13] that $k_{0}(\mu, a, b)$ is increasing in $\mu$ and $a$, and is decreasing in $b$. More precisely,

$$
\begin{gathered}
\mu_{1} \geq \mu_{2}, a_{1} \geq a_{2} \text { and } b_{1} \leq b_{2} \text { imply } k_{0}\left(\mu_{1}, a_{1}, b_{1}\right) \geq \\
k_{0}\left(\mu_{2}, a_{2}, b_{2}\right),
\end{gathered}
$$

with strict inequality holding when $\left(\mu_{1}, a_{1}, b_{1}\right) \neq\left(\mu_{2}, a_{2}, b_{2}\right)$. It is can also easily shown that $k_{0}(\mu, a, b)$ is a continuous function. Using the function $k_{0}(\mu, a, b)$, we have the following estimates for the spreading speed of $h(t)$.

Since that the assumption (3) holds, we can define

$$
\begin{aligned}
\alpha^{\infty} & :=\varlimsup_{\lim }+\infty \alpha(\mathrm{r}) \leq \kappa_{2}, \alpha_{\infty}:=\underline{\lim }_{r \rightarrow \infty} \alpha(r) \geq \kappa_{1} ; \\
\beta^{\infty} & :=\varlimsup_{\lim _{\mathrm{r} \rightarrow \infty}} \beta(\mathrm{r}) \leq \kappa_{2}, \beta_{\infty}:=\underline{\lim }_{r \rightarrow \infty} \beta(r) \geq \kappa_{1} .
\end{aligned}
$$

Theorem 4.1. If $h_{\infty}=\infty$, then

$$
k_{0}\left(\mu, \alpha_{\infty}, \beta^{\infty} \delta^{-1}\right) \leq \liminf _{t \rightarrow \infty} \frac{h(t)}{t} \leq \limsup _{t \rightarrow \infty} \frac{h(t)}{t} \leq k_{0}\left(\mu, \alpha^{\infty}, \beta_{\infty}\left(M_{1}+\delta\right)^{-1}\right)
$$

Proof. Since $(u, v, h)$ satisfies

$$
\begin{cases}v_{t}-\Delta v=v\left(\alpha(r)-\frac{\beta(r) v}{u+\delta}\right) \leq v\left(\alpha(r)-\frac{\beta(r)}{M_{1}+\delta} v\right), & t>0,0<r<h(t) \\ v_{r}(t, 0)=v(t, h(t))=0, & t>0 \\ h^{\prime}(t)=-\mu v_{r}(t, h(t))=0, & t>0 \\ h(0)=h_{0}, v(0, r)=v_{0}(r)>0, & 0 \leq r \leq h_{0}\end{cases}
$$

then $(v, h)$ is a lower solution to the following problem

$$
\begin{cases}\bar{v}_{t}-\Delta \bar{v}=\bar{v}\left(\alpha(r)-\beta(r)\left(M_{1}+\delta\right)^{-1} \bar{v}\right), & t>0,0<r<\bar{h}(t), \\ \bar{v}_{r}(t, 0)=0, \bar{v}(t, \bar{h}(t))=0, & t>0, \\ \bar{h}^{\prime}(t)=-\mu \bar{v}_{r}(t, \bar{h}(t)), & t>0, \\ \bar{h}(0)=h_{0}, \bar{v}(0, r)=v_{0}(r), & r \in[0, \bar{h}(0)] .\end{cases}
$$

It follows that $\bar{h}(t) \geq h(t) \rightarrow \infty$ as $t \rightarrow \infty$. By [8], we can obtain

$$
\limsup _{t \rightarrow \infty} \frac{\bar{h}(t)}{t} \leq k_{0}\left(\mu, \alpha^{\infty}, \beta_{\infty}\left(M_{1}+\delta\right)^{-1}\right)
$$

Thus we have

$$
\limsup _{t \rightarrow \infty} \frac{h(t)}{t} \leq k_{0}\left(\mu, \alpha^{\infty}, \beta_{\infty}\left(M_{1}+\delta\right)^{-1}\right) .
$$

Similarly, it is easy to show

$$
\liminf _{t \rightarrow \infty} \frac{h(t)}{t} \geq k_{0}\left(\mu, \alpha_{\infty}, \beta^{\infty} \delta^{-1}\right) .
$$

Corollary 4.1. Assume that $h_{\infty}=\infty$, if there exist $\alpha^{*}, \beta^{*}$ such that $\alpha(r) \rightarrow \alpha^{*}, \beta(r) \rightarrow \beta^{*}$ as $r \rightarrow \infty$, then

$$
\begin{gathered}
k_{0}\left(\mu, \alpha^{*}, \beta^{*} \delta^{-1}\right) \leq \liminf _{t \rightarrow \infty} \frac{h(t)}{t} \leq \limsup _{t \rightarrow \infty} \frac{h(t)}{t} \leq \\
k_{0}\left(\mu, \alpha^{*}, \beta^{*}\left(M_{1}+\delta\right)^{-1}\right) .
\end{gathered}
$$

Remark 4.1. We notice that the asymptotic spreading speed of (2) depends on the bounds of the prey, on parameter $\mu$ and on $\alpha(r), \beta(r)$. If $\alpha^{*}=\beta^{*}=1$, then it is the corresponding estimate of the speed to Theorem 4.1 of [5]. If the coefficients $a(r), b(r), c(r), \alpha(r), \beta(r)$ are all positive constants, then the theorems of the long time behaviors for (2) all hold, such as in [5].

\section{Conclusion}

We have examined the dynamical behavior of the population $v(t, x)$ and $u(t, x)$ with spreading front $x=h(t)$ determined by (2), and also the dynamical behavior of the population $\mathrm{u}(\mathrm{t}, \mathrm{x})$ and $\mathrm{v}(\mathrm{t}, \mathrm{x})$. We have proved that for both problems, a spreading-vanishing dichotomy holds (Theorems 3.1, 3.3 and 3.4), and when spreading occurs the spreading fronts expand at a nearly constant speed for large time (Theorem 4.1 and Corollary 4.1.). These phenomena are in agreement with numerous documented observations for the spreading of species in ecology, but differ from the mathematical conclusions obtained from (2), which predicts successful spreading for all initial data.

\section{References}

[1] G. Bunting, Y. Du and K. Krakowski, Spreading speed revisited: analysis of a free boundary model, Netw. Heterog. Media, 7 (2012), 583-603.

[2] R. S. Cantrell and C. Cosner, Diffusive logistic equations with indefinite weights: population models in disrupted environments, Proc. Roy. Soc. Edinburgh Sect. A, 112 (1989), 293-318.

[3] R. S. Cantrell and C. Cosner, Spatial ecology via reactiondiffusion equations, John Wiley and Sons, 2003. 
[4] X. Chen and A. Friedman, A free boundary problem arising in a model of wound healing, SIAM J. Math. Anal., 32 (2000), 778-800.

[5] H. Cheng and R. Yuan, A free boundary problem of some Lesile-Gower predator-prey model with higher dimensional environment, submitted, (2019).

[6] H. Cheng and R. Yuan, The spreading property for a preypredator reaction-diffusion system with fractional diffusion, Frac. Calc. Appl. Anal., 18 (2015), 565-579.

[7] H. Cheng and R. Yuan, Existence and stability of traveling waves for Leslie-Gower predator-prey system with nonlocal diffusion, Discrete Contin. Dyn. Syst. Ser. A, 37 (2017), 5422-5454.

[8] Y. Du and Z. Guo, Spreading-vanishing dichotomy in a diffusive logistic model with a free boundary, II, J. Differential Equations, 250 (2011), 4336-4366.

[9] Y. Du and Z. Guo, The Stefan problem for the Fisher-KPP equation, J. Differential Equations, 253 (2012), 996-1035.

[10] Y. Du, Z. Guo and R. Peng, A diffusive logistic model with a free boundary in time-periodic environment, J. Funct. Anal., 265 (2013), 2089-2142.

[11] Y. Du and S.-B. Hsu, A diffusive predator-prey model in heterogeneous environment, J. Differential Equations, 203 (2004), 331-364.

[12] Y. Du and X. Liang, Pulsating semi-waves in periodic media and spreading speed determined by a free boundary model, Ann. Inst. H. Poincaré Anal. Non Linéaire, 32 (2015), 279-305.

[13] Y. Du and Z. Lin, Spreading-vanishing dichotomy in the diffusive logistic model with a free boundary, SIAM J. Math. Anal., 42 (2010), 377-405.

[14] Y. Du and Z. Lin, The diffusive competition model with a free boundary: invasion of a superior or inferior competitor, Discrete Contin. Dyn. Syst. Ser. B, 19 (2014), 3105-3132.

[15] Y. Du and B. Lou, Spreading and vanishing in nonlinear diffusion problems with free boundaries, J. Eur. Math. Soc., 17 (2015), 2673-2724.

[16] Y. Du and L. Ma, Logistic type equations on $\mathbb{R}^{n}$ by a sequeezing method involving boundary blow-up solutions, J. London Math. Soc., 64 (2001), 107-124.

[17] Y. Du, H. Matsuzawa and M. Zhou, Sharp estimate of the spreading speed determined by nonlinear free boundary problems, SIAM J. Math. Anal., 46 (2014), 375-396.

[18] A. Ducrot, Convergence to generalized transition waves for some Holling-Tanner prey-predator reaction-diffusion system, J. Math. Pures Appl., 100 (2013), 1-15.

[19] J.-S. Guo and C.-H. Wu, On a free boundary problem for a two-species weak competition system, J. Dynam. Differential Equations, 24 (2012), 873-895.

[20] J.-S. Guo and C.-H. Wu, Dynamics for a two-species competition-diffusion model with two free boundaries, Nonlinearity, 28 (2015), 1-27.

[21] D. Hilhorst, M. Iida, M. Mimura and H. Ninomiya, A competition-diffusion system approximation to the classical two-phase Stefan problem, Japan J. Indust. Appl. Math., 18 (2001), 161-180.
[22] D. Hilhorst, M. Mimura and R. Schätzle, Vanishing latent heat limit in a Stefan-like problem arising in biology, Nonlinear Anal. Real World Appl., 4 (2003), 261-285.

[23] H. Huang and M. Wang, The reaction-diffusion system for an SIR epidemic model with a free boundary, Discrete Contin. Dyn. Syst. Ser. B, 20 (2015), 2039-2050.

[24] Y. Kaneko and Y. Yamada, A free boundary problem for a reaction-diffusion equation appearing in ecology, Adv. Math. Sci. Appl., 21 (2011), 467-492.

[25] O. g. A. Ladyzhenskaia, V. A. Solonnikov and N. N. Ural'tseva, Linear and quasi-linear equations of parabolic type, American Mathematical Soc., 1968.

[26] Z. Lin, A free boundary problem for a predator-prey model, Nonlinearity, 20 (2007), 1883-1892.

[27] Y. Liu, Z. Guo, M. E. Smaily and L. Wang, A Leslie-Gower predator-prey model with a free boundary, Discrete Contin. Dyn. Syst. Ser. S, 12 (2019), 2063-2084.

[28] M. Mimura, Y. Yamada and S. Yotsutani, A free boundary problem in ecology, Japan J. Appl. Math., 2 (1985), 151-186.

[29] M. Mimura, Y. Yamada and S. Yotsutani, Stability analysis for free boundary problems in ecology, Hiroshima Math. J., 16 (1986), 477-498.

[30] M. Mimura, Y. Yamada and S. Yotsutani, Free boundary problems for some reaction-diffusion equations, Hiroshima Math. J., 17 (1987), 241-280.

[31] R. Peng and X. Q. Zhao, The diffusive logistic model with a free boundary and seasonal succession, Discrete Contin. Dyn. Syst., 33 (2013), 2007-2031.

[32] M. Wang, On some free boundary problems of the preypredator model, J. Differential Equations, 256 (2014), 33653394.

[33] M. Wang, Spreading and vanishing in the diffusive preypredator model with a free boundary, Commun. Nonlinear Sci. Numer. Simul., 23 (2015), 311-327.

[34] M. Wang, A diffusive logistic equation with a free boundary and sign-changing coefficient in time-periodic environment, J. Funct. Anal., 270 (2016), 483-508.

[35] M. Wang and Y. Zhang, Two kinds of free boundary problems for the diffusive prey-predator model, Nonlinear Anal. Real World Appl., 24 (2015), 73-82.

[36] M. Wang and J. Zhao, Free boundary problems for a LotkaVolterra competition system, J. Dynam. Differential Equations, 26 (2014), 655-672.

[37] Y. Zhang and M. Wang, A free boundary problem of the ratiodependent prey-predator model, Appl. Anal., 94 (2015), 21472167.

[38] J. Zhao and M. Wang, A free boundary problem of a predatorprey model with higher dimension and heterogeneous environment, Nonlinear Anal., 16 (2014), 250-263.

[39] P. Zhou and D. Xiao, The diffusive logistic model with a free boundary in heterogeneous environment, J. Differential Equations, 256 (2014), 1927-1954. 